\title{
Serum Vitamin D and Long-term Outcomes of Benign Paroxysmal Positional Vertigo
}

\author{
Gu II Rhim (D) \\ One Otorhinolaryngology Clinic, Paju, Korea
}

Objectives. The purpose of the present study was to examine the effect of serum vitamin D concentrations on the longterm recurrence rates of benign paroxysmal positional vertigo (BPPV) patients.

Methods. The present study was conducted with patients diagnosed with BPPV from June 2014 to April 2016. Whether the patients' sex, age, types and locations of semicircular canals, diabetes, hypertension, hyperlipidemia, and vitamin D concentrations affect their recurrence rates was examined using Pearson chi-square tests, independent samples $t$-tests and Cox proportional hazards regression analyses. The effects of vitamin D concentrations on long-term recurrence rates were examined using Kaplan-Meier estimates and log-rank tests.

Results. The recurrence rates obtained with Kaplan-Meier estimates were $18 \%$ and $50 \%$ at 12 months and 24 months, respectively. When the patients were divided into groups with vitamin $\mathrm{D}$ concentrations of $<10 \mathrm{ng} / \mathrm{mL}$ and $\geq 10 \mathrm{ng} / \mathrm{mL}$ and the recurrence rates of the groups were compared, the difference was statistically significant $(P=0.040)$. In addition, when the patients were divided into groups with vitamin $\mathrm{D}$ concentrations of $<15 \mathrm{ng} / \mathrm{mL}$ and $\geq 15 \mathrm{ng} / \mathrm{mL}$ and the recurrence rates of the groups were compared, the difference was statistically quite significant $(P=0.017)$. In a Cox regression model, variables such as age, sex, the types and locations of semicircular canals, hypertension, diabetes, hyperlipidemia, and 25-hydroxy vitamin D did not significantly affect recurrence.

Conclusion. The present study investigated the recurrence rates of BPPV in patients for a long time without limiting the sex, age, or locations of semicircular canals and it could be seen that serum vitamin D concentrations significantly affected the recurrence of BPPV.

Keywords. Benign Paroxysmal PositionalVertigo; Vitamin D Deficiency; Recurrence; Kaplan-Meier Estimate

\section{INTRODUCTION}

Benign paroxysmal positional vertigo (BPPV) is known to be a disease in which otoconia, which has dropped out of the maculae of the utricle and saccule, enters the semicircular canal or adheres to the cupula and causes clinical symptoms according to position changes.

Treatment with a particle repositioning maneuver (PRM) is

\footnotetext{
- Received March 18, 2018

Revised September 5, 2018

Accepted November 21, 2018

- Corresponding author: Gu Il Rhim

One Otorhinolaryngology Clinic, 1 Hugok-ro, Paju 10924, Korea

Tel: +82-31-942-1275, Fax: +82-31-942-1278

E-mail: guzi9170@hanmail.net
}

the standard treatment, but the 1-year recurrence rate is about $20 \%$ and recurrence rates of $30 \%-50 \%$ are shown within 3-5 years [1-3]. Age, otologic diseases, and the type of canal are factors that affect the recurrence of idiopathic BPPV, and recently, the association between idiopathic BPPV and metabolic disease/ vitamin $\mathrm{D}$ has been attracting attention $[4,5]$.

Otoconia are composed of crystals of calcium carbonate $\left(\mathrm{CaCO}_{3}\right)$ and glycoproteins, which are its main components, and is connected with protein fibers on hair cells. The formation, development, and degeneration processes of otoconia are known to be regulated by the active calcium metabolic process of the vestibular organ, in contrast to the low calcium concentration in the endolymph. Some study results have indicated that vitamin D-related epithelial calcium channel proteins are involved in the calcium metabolism of the vestibular organ [6].

Copyright (C) 2019 by Korean Society of Otorhinolaryngology-Head and Neck Surgery.

This is an open-access article distributed under the terms of the Creative Commons Attribution Non-Commercial License (http://creativecommons.org/licenses/by-nc/4.0)

which permits unrestricted non-commercial use, distribution, and reproduction in any medium, provided the original work is properly cited. 
Recently, there have been studies indicating that osteoporosis and osteopenia, which are representative calcium metabolic diseases, are associated with BPPV [7-9], and there have been reports indicating that patients with severely recurring BPPV had a quite serious vitamin $D$ deficiency, along with study results indicating that BPPV patient groups have lower vitamin D concentrations [10-12]. The present study was conducted to examine the effect of serum 25-hydroxy $(25-\mathrm{OH})$ vitamin D on the long-term recurrence of BPPV.

\section{MATERIALS AND METHODS}

\section{Study subjects}

The subjects of the present study were selected from among patients diagnosed with BPPV at the One Otorhinolaryngology Clinic (Paju, Korea) from June 2014 to April 2016. In order to select the subjects for this study, 520 patients with BPPV were examined. Patient information on age, gender, comorbidities (diabetes, hypertension, and hyperlipidemia), $25-\mathrm{OH}$ vitamin $\mathrm{D}$ concentrations, and the type and location of semicircular canals involved in the initial BPPV attacks were reviewed retrospectively. All patients had blood tests done upon first presentation, regardless of recurrence. As a serum test, the $25-\mathrm{OH}$ vitamin D concentration was measured using chemiluminescence immunoassay (Centaur; Siemens Healthineers, Erlangen, Germany). The reference range of the serum test for $25-\mathrm{OH}$ vitamin $\mathrm{D}$ concentrations used in this study was above $4 \mathrm{ng} / \mathrm{mL}$. The serum vitamin D level was classified as normal $(>20 \mathrm{ng} / \mathrm{mL})$, insufficient $(10-20 \mathrm{ng} / \mathrm{mL})$, or deficient $(<10 \mathrm{ng} / \mathrm{mL}$ ). Whether the patients had any hypertension, diabetes, or hyperlipidemia was checked through questionnaire surveys.

Among the patients, those with ear diseases such as homonymous Meniere disease, vestibular neuritis, and chronic otitis media, traumatic BPPV patients, multiple canal BPPV patients, patients who were taking vitamin $\mathrm{D}$ when they were first diagnosed with BPPV, and patients treated with vitamin D during their follow-up periods were excluded. Three hundred thirtytwo patients were retrospectively followed up until October 2017. Patient information was obtained from patient records, and direct telephone calls to patients were made to ensure the accuracy of the recurrence data. Informed consents from the pa-

\section{H I G H L I G G H T S}

- The 24-month recurrence rate of benign paroxysmal positional vertigo was $50 \%$.

- The recurrence rate was higher in the low vitamin D supplement group.

- However, vitamin D supplement did not significantly decrease the recurrence rate in a multivariate model. tients are not needed because this is a retrospective study.

\section{Clinical diagnosis of BPPV}

All patients underwent physical examinations using pure tone audiometry, videonystagmography, and video Frenzel glasses when they first visited the hospital and were diagnosed and treated by one physician. Dix-Hallpike, supine roll, and supine straight head hanging tests were performed to determine lesion locations [13]. For posterior semicircular canal-BPPV, the DixHallpike test was positive if nystagmus was recorded with appropriate positioning, latency, duration, and fatigability. Lateral semicircular canal-BPPV (LC-BPPV) was diagnosed by horizontal direction-changing positional nystagmus concurrent with vertigo triggered by the supine roll test. LC-BPPV was classified as canalolithiasis or cupulolithiasis according to the direction of the nystagmus as horizontal geotropic and apogeotropic nystagmus, respectively. A down-beating nystagmus accompanied by a less pronounced torsional component to the affected side suggested anterior semicircular canal-BPPV by supine straight head hanging tests or Dix-Hallpike test.

For treatment, the modified Epley method was used in the case of canalolithiasis of the posterior semicircular canal, the Barbecue rotation method was used in the case of canalolithiasis of the lateral semicircular canal, and the Appiani and Gufoni methods were used in the case of lateral-canal cupulolithiasis. The Appiani maneuver rotates the head upward in the third therapeutic position and aims to reposition the canalith within the anterior arm or the cupulolith on the canal side of the cupula. On the other hand, the original Gufoni maneuver, introduced by Mauro Gufoni in 1998, should be performed in cases where the cupulolith is attached on the utricular side of the cupula. The patient lies down quickly on the side with the unaffected ear and remains in this position for 1 to 2 minutes until the evoked nystagmus subsides. The head is then quickly rotated $45^{\circ}$ toward the floor. The Yacovino method was used in the case of canalolithiasis of the anterior semicircular canal. The treatment was performed with a PRM one or two times a day with intervals of 2-3 days. The criteria for a complete cure were determined to be the disappearance of symptoms and the disappearance of nystagmus in physical examinations. The criterion for recurrence was a diagnosis of BPPV based on the confirmation of nystagmus with video Frenzel glasses due to dizziness that occurred again at least one month after the complete cure.

\section{Statistical analysis}

Data were expressed as median (interquartile range [IQR]). The risk factors associated with the recurrence of BPPV were compared using Pearson chi-square test and independent samples $t$ tests. The recurrence until the end of follow-up was compared using Kaplan-Meier estimates and log-rank tests, with cases censored at the end of the follow-up. Since many studies announced results indicating that vitamin $\mathrm{D}$ deficiency $(<10 \mathrm{ng} / \mathrm{mL})$ affects 
BPPV $[10,11]$, and studies on the treatment of BPPV with vitamin $\mathrm{D}$ included those conducted with deficiency patients [12] in the present study, a vitamin $\mathrm{D}$ concentration of $10 \mathrm{ng} / \mathrm{mL}$ was used as a cutoff value. In addition, since all the blood concentrations of BPPV recurrence groups and non-recurrence groups were observed to be at least $10 \mathrm{ng} / \mathrm{mL}$ in other studies [5,11], another classification was considered necessary for studies of treatment, etc. Therefore, $15 \mathrm{ng} / \mathrm{mL}$ was used as another cutoff value.

In addition, a multivariate Cox proportional hazards regression analysis was performed to compare recurrence by age, sex, 25-OH vitamin D concentration, comorbidities (diabetes, hypertension, and hyperlipidemia), and the location and type of semicircular canals involved. Statistical analyses were conducted using IBM SPSS ver. 19.0 (IBM Corp., Armonk, IL, USA). Results with a $P$-value below 0.05 were regarded as statistically significant.

\section{RESULTS}

The median age of all 332 patients (male:female $=100: 232$ ) was 50 years (IQR, 24 years), and the number of female patients was 2.3 times larger than the number of male patients at 100 ( 50 years [IQR, 21 years]; range, 13 to 83 years) while the number of female patients was 232 (49 years [IQR, 24 years]; range, 11 to 88 years). The median (IQR) serum 25-OH vitamin D concentration of all patients was $14.7(10.4) \mathrm{ng} / \mathrm{mL}$, while that of male patients was 15.7 (8.9) $\mathrm{ng} / \mathrm{mL}$ (range, 4 to $33.1 \mathrm{ng} / \mathrm{mL}$ ) and that of female

Table 1. Clinical characteristics of no recurrence group and recurrence group in benign paroxysmal positional vertigo

\begin{tabular}{lccc}
\hline Variable & $\begin{array}{c}\text { No recurrence } \\
(\mathrm{n}=279)\end{array}$ & $\begin{array}{c}\text { Recurrence } \\
(\mathrm{n}=53)\end{array}$ & $P$-value \\
\hline Age $(\mathrm{yr})$ & $50(27)$ & $47(26)$ & $0.653^{\mathrm{b})}$ \\
Female sex & $189(68)$ & $43(81)$ & 0.051 \\
Location & & & \\
$\quad$ Posterior & 63 & 12 & 0.104 \\
Lateral & 211 & 37 & 0.992 \\
Anterior & 5 & 4 & 0.372 \\
Type & & & \\
Canalolithiasis & 161 & 29 & 0.770 \\
Cupulolithiasis & 92 & 20 & 0.687 \\
$\quad$ Both & 26 & 4 & 0.502 \\
Hypertension & $72(26)$ & $16(30)$ & 0.508 \\
Hyperlipidemia & $37(13)$ & $12(23)$ & 0.078 \\
Diabetics & $23(8)$ & $5(9)$ & 0.775 \\
25-OH vitamin D (ng/mL) & $14.9(13.9)$ & $14.1(13.5)$ & $0.450^{\text {b) }}$ \\
\hline
\end{tabular}

Values are presented as median (interquartile range) or number (\%). 25-OH, 25-hydroxy.

a) Result of the Pearson chi-square tests for participants in the recurrence and no-recurrence groups except age and vitamin D. ${ }^{b}$ Result of the independent samples t-tests. patients was $14.1(10.8) \mathrm{ng} / \mathrm{mL}$ (range, 4 to $51.5 \mathrm{ng} / \mathrm{mL}$ ).

Table 1 shows the clinical characteristics of the recurrence group and the non-recurrence group. Fifty-three of the 332 patients $(16 \%)$ suffered a recurrence during the follow-up periods. The recurrence group and the non-recurrence group showed no statistically significant difference in age, location and type of semicircular canals, hypertension, diabetes, and serum vitamin $\mathrm{D}$ (25-OH vitamin $\mathrm{D})$ concentrations. The two groups showed differences in the number of females $(P=0.051$, Pearson chisquare tests) and hyperlipidemia $(P=0.078$, Pearson chi-square tests), but the differences were not statistically significant.

Fig. 1 shows the long-term recurrence rates of 332 patients as Kaplan-Meier estimates. The 12-month recurrence rate was $18 \%$ and the 24-month recurrence rate was $50 \%$. With regard to the Kaplan-Meier estimates, the patients were divided into a group with serum vitamin $\mathrm{D}$ concentrations lower than $10 \mathrm{ng} / \mathrm{mL}$ and a group with none lower than $10 \mathrm{ng} / \mathrm{mL}$. The 12 -month and 24-month recurrence rates of the group with serum vitamin D concentrations lower than $10 \mathrm{ng} / \mathrm{mL}$ were $21 \%$ and $57 \%$, respectively, while those of the group with none lower than $10 \mathrm{ng} / \mathrm{mL}$ were $17 \%$ and $45 \%$, respectively, and the differences were statistically significant $(P=0.040)$ (Fig. 2).

In addition, the patients were divided into a group with serum vitamin $\mathrm{D}$ concentrations lower than $15 \mathrm{ng} / \mathrm{mL}$ and a group with none lower than $15 \mathrm{ng} / \mathrm{mL}$. The 12-month and 24-month recurrence rates of the group with serum vitamin $\mathrm{D}$ concentrations lower than $15 \mathrm{ng} / \mathrm{mL}$ were $18 \%$ and $60 \%$, respectively, while those of the group with none lower than $15 \mathrm{ng} / \mathrm{mL}$ were $18 \%$ and $40 \%$, respectively, and the differences were statistically quite significant $(P=0.017)$ (Fig. 3$)$.

A Cox proportional hazard model was used to analyze the effect of the variables shown in Table 1 on the recurrence of BPPV over a long period of time. In a Cox regression model examined

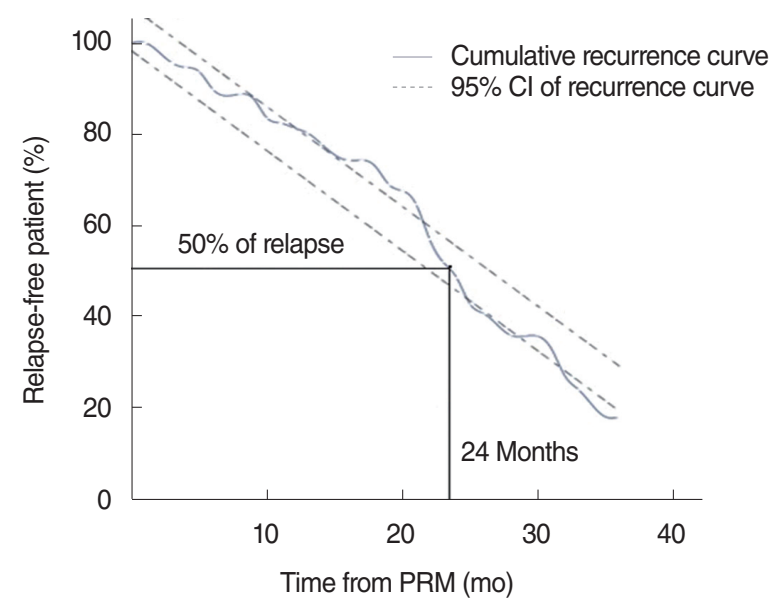

Fig. 1. Recurrence rate of benign paroxysmal positional vertigo. The percentage of patients who had recurrence is $50 \%$ at 24 months by Kaplan-Meier estimate. Cl, confidence interval; PRM, particle repositioning maneuver. 


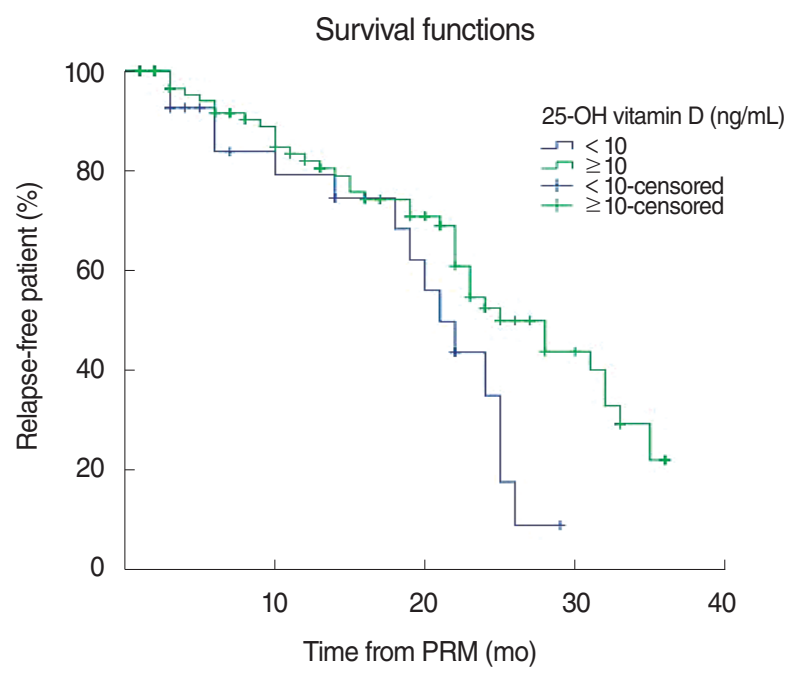

Fig. 2. Kaplan-Meier estimate for recurrence among patients with 25-hydroxy $(25-\mathrm{OH})$ vitamin D concentration $<10 \mathrm{ng} / \mathrm{mL}$ and those with $\geq 10 \mathrm{ng} / \mathrm{mL}$ after initial particle repositioning maneuver (PRM). The recurrent rate is significantly higher in patients with $25-\mathrm{OH}$ vitamin D concentration $<10 \mathrm{ng} / \mathrm{mL}(P=0.040$, log-rank test).

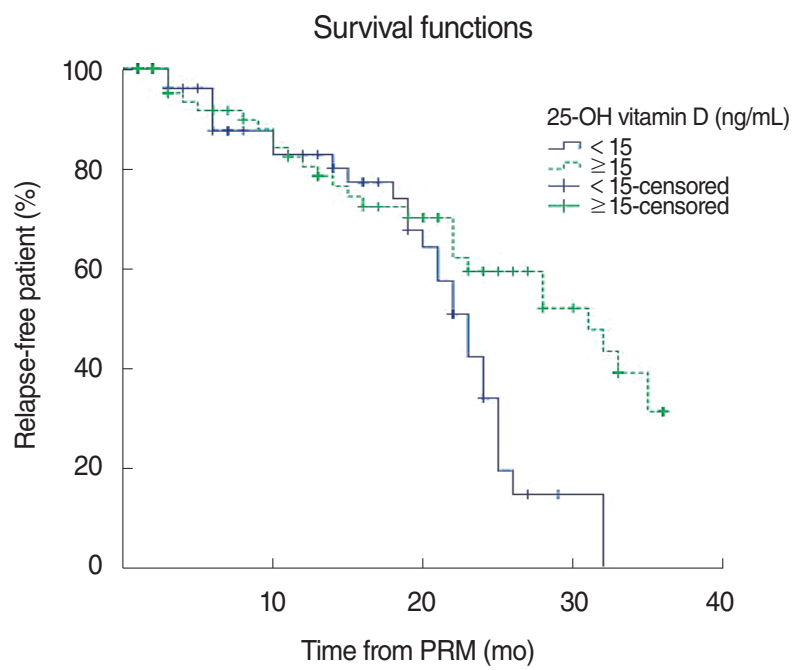

Fig. 3. Kaplan-Meier estimate for recurrence among patients with 25 -hydroxy $(25-\mathrm{OH})$ vitamin D concentration $<15 \mathrm{ng} / \mathrm{mL}$ and those with $\geq 15 \mathrm{ng} / \mathrm{mL}$ after initial particle repositioning maneuver (PRM). The recurrent rate is significantly higher in patients with $25-\mathrm{OH}$ vitamin D concentration $<15 \mathrm{ng} / \mathrm{mL}(P=0.017$, log-rank test).

using multivariate Cox proportional hazard regression analyses, variables such as age (odds ratio [OR], $0.995 ; 95 \%$ confidence interval [CI], 0.973 to 1.018; $P=0.691$ ), female sex (OR, 0.665; CI, 0.322 to $1.376 ; P=0.272)$, posterior canal $(P=0.193)$, lateral canal (OR, 0.371; CI, 0.110 to $1.257 ; P=0.111$ ), anterior canal (OR, 0.352; CI, 0.112 to $1.103 ; P=0.073$ ), canalolithiasis type $(P=0.564)$, cupulolithiasis type (OR, 0.621 ; CI, 0.208 to 1.860 ; $P=0.395)$, both type (OR, $0.832 ; \mathrm{CI}, 0.272$ to $2.543 ; P=0.746)$, hypertension (OR, 1.650; $\mathrm{CI}, 0.758$ to $3.590 ; P=0.207)$, hyper-
Table 2. Multivariate Cox proportional hazards analysis of variable of benign paroxysmal positional vertigo recurrence

\begin{tabular}{lcccc}
\hline Variable & $\mathrm{B}$ & $\mathrm{SE}$ & $P$-value & $\mathrm{OR}(95 \% \mathrm{Cl})$ \\
\hline Age & -0.005 & 0.012 & 0.691 & $0.995(0.973-1.018)$ \\
Female sex & -0.408 & 0.371 & 0.272 & $0.665(0.322-1.376)$ \\
Location & & & & - \\
$\quad$ Posterior & - & - & 0.193 & - \\
$\quad$ Lateral & -0.991 & 0.622 & 0.111 & $0.371(0.110-1.257)$ \\
$\quad$ Anterior & -1.044 & 0.583 & 0.073 & $0.352(0.112-1.103)$ \\
Type & & & & - \\
Canalolithiasis & - & - & 0.564 & $0.621(0.208-1.860)$ \\
$\quad$ Cupulolithiasis & -0.476 & 0.559 & 0.395 & $0.832(0.272-2.543)$ \\
$\quad$ Both & -0.184 & 0.570 & 0.746 & 0.830 \\
Hypertension & 0.501 & 0.397 & 0.207 & $1.650(0.758-3.590)$ \\
Hyperlipidemia & 0.264 & 0.375 & 0.481 & $1.303(0.624-2.718)$ \\
Diabetics & -0.150 & 0.545 & 0.783 & $0.861(0.296-2.503)$ \\
25-OH vitamin D & -0.024 & 0.023 & 0.279 & $0.976(0.934-1.020)$ \\
\hline
\end{tabular}

$\mathrm{B}$, unstandardized coefficient; SE, standard error; OR, odds ratio; $\mathrm{Cl}$, confidence interval; 25-OH, 25-hydroxy.

lipidemia (OR, 1.303; CI, 0.624 to 2.718; $P=0.481$ ), diabetics (OR, 0.861; CI, 0.296 to $2.503 ; P=0.783$ ) and $25-\mathrm{OH}$ vitamin $\mathrm{D}$ (OR, 0.976; CI, 0.934 to $1.020 ; P=0.279)$ did not significantly affect recurrence. As a continuous variable, vitamin $\mathrm{D}$ is not a statistically significant variable for recurrence. According to regression coefficients, vitamin $\mathrm{D}$ has a coefficient of -0.024 . This indicates that the higher the vitamin $\mathrm{D}$ level, the lower the recurrence rate (Table 2).

In conclusion, according to Pearson chi-square tests, independent samples $t$-tests and the Cox proportional hazards model, none of age, sex, location and type of semicircular canal, hypertension, diabetes, hyperlipidemia, and vitamin $\mathrm{D}$ concentration statistically significantly affected the recurrence rates.

The recurrence rates obtained using Kaplan-Meier estimates and log-rank tests were significantly different between the group with vitamin $\mathrm{D}$ concentrations lower than $10 \mathrm{ng} / \mathrm{mL}$ and the group with vitamin $\mathrm{D}$ concentrations not lower than $10 \mathrm{ng} / \mathrm{mL}$ $(P=0.040)$. And the recurrence rates using the estimates and tests were more statistically significantly different between the group with vitamin $\mathrm{D}$ concentrations lower than $15 \mathrm{ng} / \mathrm{mL}$ and the group with vitamin $\mathrm{D}$ concentrations not lower than $15 \mathrm{ng} / \mathrm{mL}$ $(P=0.017)$.

\section{DISCUSSION}

The long-term recurrence rates of BPPV are generally known to reach $30 \%-50 \%$ between 3 and 5 years $[2,3]$. The factors known to be associated with recurrence include comorbid ear diseases, age, female, lateral canal, the number of PRMs, chronic diseases, and vitamin $\mathrm{D}$ deficiency $[1,3,5]$.

In a 5-year long-term recurrence rate study conducted with 118 patients with posterior semicircular canal BPPV, Kansu et 
al. [14] reported that the disease recurred in one third of the patients and that head trauma and Meniere disease affected the recurrence. In a long-term recurrence rate study conducted with 125 patients with posterior semicircular canal BPPV through questionnaires, Brandt et al. [3] reported that the 10 -year recurrence rate was $50 \%$ and $80 \%$ of the recurrent cases occurred within a year. Perez et al. [15] reported that based on the results of observation of $69 \mathrm{BPPV}$ patients over 5 years, BPPV recurred within 6 months in $50 \%$ of the patients and multiple canals and anterior canals affected the recurrence. In the present study, the recurrence rate was shown to be $50 \%$ for 24 months in KaplanMeier analysis.

In this study, the number of women was higher in the recurrence group $(P=0.051)$, but the difference was not statistically significant. The locations and types of semicircular canals did not affect the recurrence rate in the present study. There were studies indicating that diabetes and hypertension affected the recurrence of BPPV $[4,16]$. However, the mean age of the subject groups was at least 60 years in those studies, while the median age of subjects in the present study was 50 years and hypertension $(P=0.508)$ or diabetes $(P=0.775)$ did not affect the recurrence rate. Patients with hyperlipidemia were more frequent in the recurrence group $(P=0.078)$, but the difference was not statistically significant. In general, the prevalence of BPPV among women was twice as high as that among men, and this difference is explained with differences in sex hormones in many cases. Therefore, in the beginning, many studies on the association between osteoporosis and BPPV were conducted with postmenopausal women with drastic changes in sex hormones $[7,8]$.

Vitamin D is an important factor in calcium metabolism and is already a well-known factor associated with bone metabolism such as osteoporosis. It is already well known that vitamin D deficiency in older adults increases the risk of fracture and vitamin D supplementation therapy reduces the risk of fracture $[17,18]$. In some studies on the metabolism of calcium carbonate and glycoprotein, which are the main components of otoconia, findings indicated that vitamin $\mathrm{D}$ is involved in calcium metabolic processes and vitamin D deficiency affects BPPV development [6,11].

To review studies of BPPV using vitamin D as a continuous variable, Jeong et al. [10] stated that vitamin $\mathrm{D}$ insufficiency $(P=0.025)$ and deficiency $(P<0.01)$ were associated with BPPV. Talaat et al. [11] showed results indicating that the vitamin $D$ concentration $(19.5 \mathrm{ng} / \mathrm{mL})$ of the control group was significantly different from that of the non-recurrent BPPV group (16.0 $\mathrm{ng} / \mathrm{mL})$, and that of the recurrent BPPV group $(11.9 \mathrm{ng} / \mathrm{mL})$. And a study conducted by Rhim [5] showed results indicating that the vitamin $\mathrm{D}$ concentration of the non-recurrence group $(16.6 \mathrm{ng} / \mathrm{mL})$ was significantly different from that of the recurrence group (13.6 $\mathrm{ng} / \mathrm{mL}$ ). Several studies implemented vitamin D supplementation therapy in an effort to reduce the recurrence rates of BPPV patients with vitamin D deficiency. Buki et al. [12] reported that when vitamin D supplementation therapy was implemented on four vitamin D deficiency patients with histories of chronically recurrent severe vertigo, the recurrence decreased. Talaat et al. [19] reported that after vitamin D supplementation therapy was implemented on 28 posterior semicircular canal BPPV patients with vitamin D deficiency for three months, its recurrence significantly decreased compared to the control group for the follow-up period of 18 months. In the present study, patients were divided into many groups with different vitamin D serum concentrations and the recurrence rates of the groups were compared. The vitamin D concentrations of the non-recurrence group and recurrence group was 16.0 and $11.9 \mathrm{ng} / \mathrm{mL}$ in the studies by Talaat et al. [11] and 16.6 and 13.6 $\mathrm{ng} / \mathrm{mL}$ in the studies by Rhim [5]. Since all the blood concentrations of BPPV recurrence groups and non-recurrence groups were observed to be at least $10 \mathrm{ng} / \mathrm{mL}$, a more detailed classification seemed to be required than a general serum classification.

However, when the patients were divided into a group with vitamin $\mathrm{D}$ concentrations lower than $10 \mathrm{ng} / \mathrm{mL}$ and a group with none lower than $10 \mathrm{ng} / \mathrm{mL}$ and the recurrence rates were compared, the recurrence rate of the group with vitamin D concentrations lower than $10 \mathrm{ng} / \mathrm{mL}$ was statistically significantly higher $(P=0.040)$. In addition, when the patients were divided into a group with vitamin $\mathrm{D}$ concentrations lower than $15 \mathrm{ng} / \mathrm{mL}$ and a group with none lower than $15 \mathrm{ng} / \mathrm{mL}$ and the recurrence rates were compared, the recurrence rate of the group with vitamin D concentrations lower than $15 \mathrm{ng} / \mathrm{mL}$ was statistically significantly higher $(P=0.017)$.

This is the first study to investigate the long-term recurrence rates of BPPV patients with vitamin D deficiency. However, the present study has the following disadvantages. The limitations are that it is a retrospective study conducted with medical records and telephone interviews and involves the risk of information bias due to the uncertainty of information collection and dependence on medical records. In addition, careful attention should be paid to the analysis and interpretation of factors influencing survival analysis, since Kaplan-Meier survival analysis is either incomplete censoring, artificially initiates and terminates study observations, and observes only survival at a certain point in time.

This study investigated the recurrence rates of BPPV patients for a long period of time without limiting the sex, age, or locations of semicircular canals, and it could be seen that serum vitamin D concentrations significantly affect the recurrence of BPPV. Given the results of the present study, treating patients with serum vitamin $\mathrm{D}$ concentrations lower than $15 \mathrm{ng} / \mathrm{mL}$ should be more helpful in the reduction of the recurrence rate than treating patients with blood vitamin $\mathrm{D}$ concentrations lower than $10 \mathrm{ng} / \mathrm{mL}$ as was done in previous studies. 


\section{CONFLICT OF INTEREST}

No potential conflict of interest relevant to this article was reported.

\section{ORCID}

Gu Il Rhim https://orcid.org/0000-0002-6182-9184

\section{REFERENCES}

1. Tanimoto H, Doi K, Nishikawa T, Nibu K. Risk factors for recurrence of benign paroxysmal positional vertigo. J Otolaryngol Head Neck Surg. 2008 Dec;37(6):832-5.

2. Sakaida M,Takeuchi K, Ishinaga H, Adachi M, Majima Y. Long-term outcome of benign paroxysmal positional vertigo. Neurology. 2003 May;60(9):1532-4.

3. Brandt T, Huppert D, Hecht J, Karch C, Strupp M. Benign paroxysmal positioning vertigo: a long-term follow-up (6-17 years) of 125 patients.Acta Otolaryngol. 2006 Feb;126(2):160-3.

4. De Stefano A, Dispenza F, Suarez H, Perez-Fernandez N, ManriqueHuarte R, Ban JH, et al. A multicenter observational study on the role of comorbidities in the recurrent episodes of benign paroxysmal positional vertigo. Auris Nasus Larynx. 2014 Feb;41(1):31-6.

5. Rhim GI. Serum vitamin D and recurrent benign paroxysmal positional vertigo. Laryngoscope Investig Otolaryngol. 2016 Oct;1(6): 150-3.

6. Lundberg YW, Zhao X, Yamoah EN. Assembly of the otoconia complex to the macular sensory epithelium of the vestibule. Brain Res. 2006 May;1091(1):47-57.

7. Vibert D, Kompis M, Hausler R. Benign paroxysmal positional vertigo in older women may be related to osteoporosis and osteopenia. Ann Otol Rhinol Laryngol. 2003 Oct;112(10):885-9.
8. Yamanaka T, Shirota S, Sawai Y, Murai T, Fujita N, Hosoi H. Osteoporosis as a risk factor for the recurrence of benign paroxysmal positional vertigo. Laryngoscope. 2013 Nov;123(11):2813-6.

9. Jeong SH, Choi SH, Kim JY, Koo JW, Kim HJ, Kim JS. Osteopenia and osteoporosis in idiopathic benign positional vertigo. Neurology. 2009 Mar;72(12):1069-76.

10. Jeong SH, Kim JS, Shin JW, Kim S, Lee H, Lee AY, et al. Decreased serum vitamin $\mathrm{D}$ in idiopathic benign paroxysmal positional vertigo. J Neurol. 2013 Mar;260(3):832-8.

11. Talaat HS, Abuhadied G, Talaat AS, Abdelaal MS. Low bone mineral density and vitamin $\mathrm{D}$ deficiency in patients with benign positional paroxysmal vertigo. Eur Arch Otorhinolaryngol. 2015 Sep;272(9): 2249-53.

12. Buki B, Ecker M, Junger H, Lundberg YW.Vitamin D deficiency and benign paroxysmal positioning vertigo. Med Hypotheses. 2013 Feb; 80(2):201-4.

13. von Brevern $M$, Bertholon $P$, Brandt $T$, Fife $T$, Imai $T$, Nuti $D$, et al. Benign paroxysmal positional vertigo: diagnostic criteria. J Vestib Res. 2015;25(3-4):105-17.

14. Kansu L, Avci S, Yilmaz I, Ozluoglu LN. Long-term follow-up of patients with posterior canal benign paroxysmal positional vertigo. Acta Otolaryngol. 2010 Sep;130(9):1009-12.

15. Perez P, Franco V, Cuesta P, Aldama P, Alvarez MJ, Mendez JC. Recurrence of benign paroxysmal positional vertigo. Otol Neurotol. 2012 Apr;33(3):437-43.

16. Kim SY, Han SH, Kim YH, Park MH. Clinical features of recurrence and osteoporotic changes in benign paroxysmal positional vertigo. Auris Nasus Larynx. 2017 Apr;44(2):156-61.

17. Dawson-Hughes B, Harris SS, Krall EA, Dallal GE. Effect of calcium and vitamin $\mathrm{D}$ supplementation on bone density in men and women 65 years of age or older. N Engl J Med. 1997 Sep;337(10):670-6.

18. Mosekilde L.Vitamin D and the elderly. Clin Endocrinol (Oxf). 2005 Mar;62(3):265-81.

19. Talaat HS, Kabel AM, Khaliel LH, Abuhadied G, El-Naga HA, Talaat AS. Reduction of recurrence rate of benign paroxysmal positional vertigo by treatment of severe vitamin D deficiency. Auris Nasus Larynx. 2016 Jun;43(3):237-41. 